\title{
Research Paper: A Comparison of the Facets of Mindfulness Among Patients With Major Depression, crossuark Social Anxiety Disorder and Healthy Individuals
}

\author{
Nasrin Alsadat Hosseini Ramaghani ${ }^{1}$, Masoud Sadeghi ${ }^{*}$, Khodayar Mehrabi \\ 1. Department of Psychology, Faculty of Literature and Humanities, Lorestan University, Khorramabad, Iran. \\ 2. Nokhbegan-e-Delfan Language School, Delfan Ministry of Education, Lorestan, Iran.
}

\begin{tabular}{|l|l}
\hline $\begin{array}{l}\text { use yourdevic to scan } \\
\text { and read thearticle online }\end{array}$ \\
$\square$
\end{tabular}

\section{Article info:}

Received: 14 May 2017

Accepted: 01 Sep. 2017
Keywords:

Mindfulness, Anxiety,

Depression, Students

\section{ABSTRACT}

Objective: There are well-established benefits of mindfulness in clinical settings, but the different roles of mindfulness facets in each disorder yet remain unclear. The purpose of the current study was to investigate between-group comparisons of the facets of mindfulness among patients with Major Depression Disorder (MDD), Social Anxiety Disorder (SAD) and healthy individuals.

Methods: The present study employs a. Using convenience sampling method, a sample composed of three groups of students with SAD ( $n=67)$, MDD $(n=51)$ and healthy $(n=81)$ completed the Five Facet Mindfulness, Beck depression, and the Social Phobia Inventory. To analyze the data, MANOVA test was conducted.

Results: Results indicated that for the observing facet, SAD group significantly had higher scores than the MDD and Healthy groups and MDD group exhibited least scores For describing, non-judge and acting with awareness facets, both SAD and MDD groups had significantly lower scores than the healthy group, while there was no significant difference between the SAD and MDD groups. For the non-reacting facet, the SAD group had significantly lower scores than the MDD and healthy groups, while there was no significant difference between MDD and healthy groups.

Conclusion: The results of this study suggest that non-judging, acting with awareness, and describing are facets within the umbrella concept of mindfulness facets, which have a common role in SAD and MDD. However, observing and non-reacting have distinct roles in the SAD and MDD. The findings of the present study will certainly have important implications for clinical health psychologists while tailoring mindfulness-based treatments for SAD and MDD patients.

\footnotetext{
* Corresponding Author:

Masoud Sadeghi, PhD

Address: Department of Psychology, Faculty of Literature and Humanities, Lorestan University, Khorramabad, Iran.

Tel: +98 (912) 8440251

E-mail:sadeghi.m@lu.ac.ir
} 


\section{Introduction}

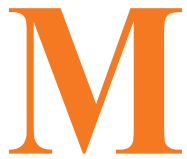

indfulness can be described as an awareness that comes from attending to current life's events in an intentional and non-judgmental way (Garland, Farb, Goldin, \& Fredrickson, 2015). In general, Mindfulness is considered as a multi-dimensional construct, except few researchers who believe it to be a single factor construct (Brown \& Ryan, 2004). Recently, the multi-dimensional conceptualization by Baer, Smith, Hopkins, Krietemeyer, and Toney (2006) has attracted the attention of many researchers.

In this model, they described five distinct factors that best depict overall mindfulness: 1) Observing, which refers to the process of attending to or noticing internal or external experiences; 2) Describing, which involves the capacity to express internal experiences (e.g. thoughts, feelings, beliefs) with words; 3) Acting with awareness, which refers to attending to current activities in the present moment without being distracted; 4) Non-judging, which involves accepting a non-evaluative viewpoint towards thoughts and feelings, and 5) Non-reacting, which consists of permitting thoughts and feelings to come and go without reacting or fixating.

The clinicians and researchers have shown much interest in mindfulness due to its contribution towards relieving mental health symptoms (Raphiphatthana, Jose, \& Kielpikowski, 2016). In fact, mindfulness-based interventions used in the clinical domain have been found to be effective in reducing a variety of psychological symptoms and disorders, particularly anxiety and depression (Bohlmeijer, Prenger, Taal, \& Cuijpers, 2010). Mindfulness-based interventions contain different techniques that are derived from the theorized facets of mindfulness. Nevertheless, it is not exactly known which techniques are most momentous to the efficiency of treatment (Holzel et al., 2011).

Moreover, although there are several studies on the relationships between particular aspects of mindfulness and the specific symptoms associated with depression and anxiety, as well as, a study on more targeted utilization of mindfulness techniques based on specific symptoms of depression and anxiety (Raphiphatthana et al., 2016), there is still no precise and clear information about the distinct roles of the components of mindfulness in anxiety disorders in comparison to the depression disorder, as well as in the groups with disorder (anxiety, depression) in comparison to the healthy group. Keeping this in view, the present study focused on investigating which components of mindfulness in one of the anxiety disorders (e.g. the Social Anxiety Disorder (SAD)) is different from both Major Depression Disorder (MDD), and healthy individuals.

SAD is characterized by a marked and persistent fear of negative evaluation in social situations (American Psychiatric Association, 2013). SAD is a chronic psychiatric disorder that is associated with poor educational and occupational achievement and interpersonal impairment (Ranta, La Greca, Kaltiala-Heino, \& Marttunen, 2016). This chronic disorder is the fourth most common psychiatric disorder with a lifetime prevalence of $10-15 \%$ and a one-year prevalence of 5-10\% (Ohayon, \& Schatzberg, 2010). SAD is most often treated by using Cognitive Behavior Therapy (CBT). Although CBT has been broadly indicated as an effective treatment for social anxiety; however, many people with social anxiety do not benefit from this treatment quality or only achieve minimal improvement (Taylor, Abramowitz, \& McKay, 2012). Thus, clinicians and researchers have recently been attracted to other methods of treatment, including mindfulness and acceptance-based interventions.

On the other hand, depression is the most prevalent of all psychiatric disorders affecting up to $25 \%$ of women and $12 \%$ of men during their lifetimes (Rubio et al., 2011). Several psychological studies have corroborated that SAD and MDD are closely related (Koyuncu et al., 2015), and share several features such as excessive negative self-focused attention (Helbig-Lang, Poels, \& Lincoln, 2016; Mor et al., 2010), rumination (Grant et al., 2014), interpersonal behaviors such as avoidance and marked interpersonal impairment (Washburn, Wilson, Roes, Rnic, \& Harkness, 2016). Since a growing number of studies has provided evidence for the effectiveness of mindfulness based interventions specifically in the context of SAD and MDD (Kocovski, Fleming, Hawley, Ho, \& Antony, 2015; Schuver, \& Lewis, 2016), highlighting the differential role of mindfulness facets in each disorder can address long-term debates about diagnostic overlap between SAD and MDD. If distinct mindfulness deficits are uniquely associated with each disorder, then this provides a basis for diminishing difficulties of diagnosis and organizing the conceptual models underlying these disorders.

As a result, such deficits might be possible candidates for treatment mechanisms in each of these disorders so that treatment protocols for each of these disorders will be consistent with possible deficits in the mindfulness facets. We assumed some hypotheses based on studies and theories of mindfulness and/or theories of anxiety 
and depression: The results of previous studies suggest that observing may be associated with heightened attention to internal anxiety cues, (e.g. Raphiphatthana et al., 2016) and socially anxious individuals have higher interceptive awareness compared to healthy groups (Deiters, Stevens, Hermann, \& Gerlach, 2013). Thus, we hypothesized that groups with SAD show higher levels of observing compared to the healthy group. In previous studies, description has been associated with alexithymia (e.g. de Bruin,Topper, Muskens, Bögels, \& Kamphuis, 2012).

Alexithymia, in turn, has been associated with MDD and SAD (e.g. Dalbudak et al., 2013). In addition, theoretically, the concept of non-judging appears to be the opposite of negative self-cognitions and self-criticism (Raphiphatthana et al., 2016), which are shared characteristics of MDD and SAD (Cox, Fleet, \& Stein, 2004). Acting with awareness has been found to be inversely associated with worry and rumination (de Bruin et al., 2012) which are associated with both social anxiety and depression (Hsu et al., 2015). Finally, the process of permitting feelings and thoughts to pass through awareness without reacting to or controlling them may be particularly beneficial for reducing negative and repetitive thought processes which are common to both social anxiety and depression (i.e. worry, rumination) (e.g. Hsu et al., 2015). Thus, we hypothesized that there are significant differences between the levels of describing, nonjudging, acting with awareness and non-reacting facets in the three groups, particularly groups with SAD and MDD show lower levels of these facets as compared to the healthy group. We also investigated the possible difference between the MDD and SAD groups based on the five facets of mindfulness.

Since, there is no precise and clear information about the distinct roles of the components of mindfulness in SAD compared with the MDD, as well as in the groups with disorder (SAD, MDD) compared with the healthy group. Further, there is a need to test psychological theories and concepts with diverse, international and Non-Western populations to specify possible cultural differences in order to address cultural applications of psychological theories and concepts. Therefore, considering these assumptions and research questions, the present study was designed to investigate the differences in the aspects of mindfulness between SAD, MDD and healthy groups.

\section{Methods}

This cross-sectional study has a causal-comparative design. Using convenience sampling method and based on the following inclusion/exclusion criteria, a sample of 67 socially anxious people, who were seeking treatment at the psychological disorders clinics of Lorestan Universities, Iran, and met the Diagnostic and Statistical Manual for Mental Disorders' fifth edition (DSM-V; American Psychiatric Association, 2013) criteria for a principal diagnosis of SAD and also got scores higher than the cutoff point (i.e. 29 points) in the Social Phobia Inventory (SPIN) (Dogaheh, 2013) were assigned to the SAD group.

Whereas, 51 depressive patients of them who met the DSM-V criteria for a principal diagnosis MDD and got scores higher than 20 in the BDI-II (Beck, Steer, \& Brown, 1996) were assigned to the MDD group. To obtain the healthy group, 116 undergraduate students, who were not seeking treatment, were screened by the SPIN and BDI-II in a way that 100 participants (from 116) who got scores lower than aforementioned scores underwent a clinical diagnostic interview by a doctorate in Clinical Psychology. Then 81 healthy persons, who did not meet the diagnostic criteria for Axis I disorders, were recruited. The SAD group included 40 females and 27 males, the MDD group included 29 females and 22 males and the healthy group included 44 females and 37 male. It should be noted that participants were excluded if they reported clinically noteworthy symptoms of psychosis, history of substance abuse, or any organic mental disorders. The informed consent was obtained from all participants before inclusion in the study and APA's Ethical Principles of Psychologists (such as mentally preparing the participants and reassuring them of the confidentiality of personal information) were strictly followed in the research work.

The measures used are the Social Phobia Inventory (SPIN), Beck depression inventory II (BDI-II), and Five Facet Mindfulness Questionnaire (FFMQ). Social Phobia Inventory (SPIN) developed by Connor et al., (2000) was used to assess social anxiety. It is a 17-item selfreport measure of avoidance and fear of a range of social situations and physiological symptoms of anxiety. The SPIN has been validated for use in clinical populations. In the present study, the reliability of 0.87 was obtained using the Cronbach's alpha coefficient. The BDI-II (Beck et al., 1996) is a multiple-choice self-report instrument designed to measure both the presence and severity of depressive symptoms. BDI-II contains 21 questions, each answer being scored on a scale value of 0 to 3 . Higher total scores indicate more severe depressive symptoms. In the present study, the Persian version of BDI-II was administered, and internal consistency with an alpha coefficient of 0.87 was obtained. 
Five Facet Mindfulness Questionnaire (FFMQ) was developed by Baer et al., (2006) and is a 39-item assessing mindfulness in daily life. Researchers mixed items from five existing mindfulness questionnaires into a single data set and performed an exploratory factor analysis. From this analysis, researchers found five component skills that contribute to mindfulness, observing, non-reactivity to inner experiences, non-judging of inner experiences, acting with awareness, and describing. Items are rated on a 5-point Likert-type scale. In the present study, Persian version of FFMQ was administered, and internal consistency with alpha coefficients ranged from 0.73 to 0.89 .

\section{Results}

Data were analyzed through Multivariate analysis of variance (MANOVA), post hoc comparisons with Tukey in the SPSS (version 23) statistical software. To use the MANOVA test, the hypothesis of equality of variances and covariance matrices, as well as the normality of data, were checked using Levene, Box's M, and Kolmogorov-Smirnov tests. Findings confirmed the assumptions $(\mathrm{P}>0.05)$. In Table 1 were indicated the descriptive statistics of the study variables.
As seen in Table 2, the results for MANOVA revealed a significant difference between various groups, namely the observing facet $(\mathrm{F}(2,196)=33.85, \mathrm{P}<0.001, \mathrm{ES}=0.25)$, the describing facet $(\mathrm{F} \quad(2,196)=57.41, \mathrm{P}<0.001$, $\mathrm{ES}=0.36)$, the non-judging facet $(\mathrm{F}(2,196)=20.29$, $\mathrm{P}<0.001, \mathrm{ES}=0.17)$, the acting with awareness facet ( $\mathrm{F}$ $(2,196)=28.60, \mathrm{P}<0.001, \mathrm{ES}=0.22)$, and the non-reacting facet $(\mathrm{F}(2,196)=53.66, \mathrm{P}<0.001, \mathrm{ES}=0.35)$.

To examine the nature of these differences in each of the groups, Post Hoc Pairwise comparisons were conducted through the Tukey test (Table 3). This analysis indicated that for the observing facet, SAD group significantly had higher scores than MDD and healthy groups and MDD group exhibiting the lowest scores. For the describing, non-judge and acting with awareness facets, both groups of SAD and MDD had significantly lower scores than the healthy group, and with no significant difference between SAD and MDD groups.

For the non-reacting facet, the SAD group had significantly lower scores than the MDD and healthy groups, and furthermore, no significant differences between the MDD and healthy groups were observed.

Table 1. The descriptive statistics of social anxiety, depression and the five facets of mindfulness by groups

\begin{tabular}{|c|c|c|c|}
\hline \multirow{2}{*}{ Measures } & \multicolumn{3}{|c|}{ Groups (Mean $\pm S D$ ) } \\
\hline & Healthy & SAD & MDD \\
\hline Observe & $24.92 \pm 6.04$ & $27.64 \pm 5.37$ & $18.54 \pm 6.78$ \\
\hline Describe & $23.11 \pm 3.65$ & $15.14 \pm 6.20$ & $15.92 \pm 4.87$ \\
\hline Non-judge & $22.80 \pm 6.25$ & $16.98 \pm 5.47$ & $17.88 \pm 6.06$ \\
\hline Act-aware & $20.23 \pm 4.61$ & $14.56 \pm 4.89$ & $15.54 \pm 5.17$ \\
\hline Non-react & $18.56 \pm 4.55$ & $11.37 \pm 3.60$ & $17.58 \pm 5.11$ \\
\hline
\end{tabular}

Table 2. Multivariate analysis of variance results

\begin{tabular}{ccccc}
\hline Variable & Sum of Squares & Mean Squares & F & Partial Eta Squared \\
\hline Observe & 2464.96 & 1232.48 & $33.85^{* *}$ & 0.25 \\
\hline Describe & 2812.01 & 1406.00 & $57.41^{* *}$ & 0.36 \\
\hline Non-judge & 1439.26 & 719.63 & $20.29^{* *}$ & 0.17 \\
\hline Act-aware & 1348.24 & 647.12 & $28.60^{* *}$ & 0.22 \\
Non-react & 2094.88 & 1047.44 & $53.66^{* *}$ & 0.35 \\
\hline Note: ${ }^{* *}$ P $<0.01$ & & & & PRetrmeAln \\
\hline
\end{tabular}


Table 3. Post hoc pairwise comparisons with Tukey

\begin{tabular}{|c|c|c|c|c|c|}
\hline Dependent Variable & (I) Group & (J) Group & Mean Difference (I-J) & Std. Error & Sig. \\
\hline & & SAD & $-2.71^{*}$ & 0.99 & 0.019 \\
\hline \multirow{3}{*}{ Observe } & Healthy & & & & \\
\hline & & MDD & $6.37^{* *}$ & 1.07 & 0.001 \\
\hline & SAD & MDD & $9.09 * *$ & 1.12 & 0.001 \\
\hline \multirow{3}{*}{ Describe } & & SAD & $7.96 * *$ & 0.81 & 0.001 \\
\hline & Healthy & MDD & $7.18^{* *}$ & 0.88 & 0.001 \\
\hline & SAD & MDD & -0.77 & 0.91 & 0.67 \\
\hline \multirow{4}{*}{ Non-judge } & & SAD & $5.81 * *$ & 0.98 & 0.001 \\
\hline & Healthy & & & & \\
\hline & & MDD & $4.92 * *$ & 1.06 & 0.001 \\
\hline & SAD & MDD & -0.89 & 1.10 & 0.69 \\
\hline \multirow{4}{*}{ Act-aware } & & SAD & $5.66 * *$ & 0.80 & 0.001 \\
\hline & Healthy & & & & \\
\hline & & MDD & $4.68 * *$ & 0.86 & 0.001 \\
\hline & SAD & MDD & -0.98 & 0.90 & 0.52 \\
\hline \multirow{4}{*}{ Non-react } & & SAD & $7.19 * *$ & 0.72 & 0.001 \\
\hline & Healthy & & & & \\
\hline & & MDD & 0.97 & 0.78 & 0.43 \\
\hline & SAD & MDD & $-6.21 * *$ & 0.82 & 0.001 \\
\hline
\end{tabular}

\section{Discussion}

The purpose of the current study was to investigate differences regarding the facets of mindfulness among patients with MDD, SAD and healthy individuals in a NonWestern population. Findings indicate that the SAD group had significantly higher scores in the observing facet than the MDD and healthy groups. Since the observing may be associated with heightened attention to internal anxiety cues, sometimes referred to as heightened interceptive awareness (Baer et al., 2008) and socially anxious individuals too have high interceptive awareness, thus observing may contribute to heightened interceptive awareness in the SAD. This suggests that in clinical practice for $\mathrm{SAD}$, concentrated observation of stressful thoughts and feelings during mindfulness practice without enough emphasis on the cultivation of the other facets (i.e. developing a nonjudgmental stance towards internal experience) may exacerbate the symptoms of social anxiety.

In addition, between-groups comparisons indicated that the MDD group had significantly lower scores in the observing facet than healthy groups. It is noteworthy that according to criteria DSM-V (American Psychiatric As- sociation, 2013), loss of concentration and attention are the clinical symptoms of depression, which could be used to explain the low scores of observing for depressed patients compared to healthy subjects. This suggests that in clinical practice for MDD, adequate emphasis should be focused on the cultivation of observing facet in this group.

The results of the present study for the describing facet are in accordance with previous reports and hypothesis. In this regard, the SAD and MDD group had significantly lower scores in the describing facet than the healthy group. But no significant difference was found between the MDD and SAD groups. In the previous studies, describing or labeling internal experiences with words has been associated with alexithymia (de Bruin et al., 2012) which is described by difficulties in describing and identifying feelings and distinguishing between emotions and physical sensation (Nowakowski, McFarlane, \& Cassin, 2013) and alexithymia has, in turn, been associated with MDD and SAD (Dalbudak et al., 2013). Therefore, it seems reasonable that the groups with $\mathrm{SAD}$ and MDD show lower levels of describing compared to the healthy group. This has implications for clinical work, where describing and labeling internal experience are 
useful in treating symptoms of anxiety and depression in MDD and SAD groups.

Similarly to an earlier hypothesis, comparisons between-groups indicated that the SAD and MDD groups had significantly lower scores in the non-judging facet than the healthy group since the appraisal of a situation or experience is central to the emotional response (Gross, 2014). Thus, by having a non-evaluative stance towards one's experience, one may be less likely to involve in a negative appraisal of the experience and also less likely to exhibit intense negative emotional reactions (Raphiphatthana et al., 2016). On the other hand, theoretically, the concept of non-judging appears to be the opposite of negative self-cognitions and self-criticism (Raphiphatthana et al., 2016) which are shared characteristics of MDD and SAD (Cox et al., 2004). Hence it seems reasonable that the groups with SAD and MDD show lower levels of non-judging compared to the healthy group. Thus, in clinical work for SAD and MDD by an emphasis on holding a non-evaluative stance towards one's experience, one may be less likely to involve in a negative appraisal of the experience for which one would be less likely to show intense negative emotional reactions.

Results also indicated that the SAD and MDD group had significantly lower scores in the acting with awareness facet than healthy group. These relationships are consistent with previous reports stating that acting with awareness is negatively related to anxiety and depression (Bohlmeijer et al., 2010), and it has also been found to be inversely associated with worry and rumination (de Bruin, 2012) which are associated with both social anxiety and depression (Hsu et al., 2015). Being aware of one's present activity may compete for attentional resources required for processing of negative repetitive thoughts (Raphiphatthana et al., 2016). Acting with awareness may decrease anxiety and depression symptoms by reducing attentional resources consumed in ruminative thinking (Bogaerde, Derom, \& De Raedt, 2011). Thus, current findings suggest that in clinical practice for SAD and MDD to reduce general symptoms of anxiety and depression, there should be enough emphasis on therapeutic techniques to improve the acting with awareness facet in these groups.

The findings consistent with previous findings in the literature (Desrosiers, Klemanski, \& Nolen-Hoeksema, 2013; de Bruin et al., 2012) indicated that the SAD group had significantly lower scores in the non-reacting facet than the MDD and healthy groups. Since the process of permitting feelings and thoughts to pass through awareness without reacting to or controlling them may be particularly beneficial for reducing negative and repetitive thought processes that are experienced by socially anxious individuals (i.e. worry, rumination) (Hsu et al., 2013), it seems reasonable that the group with $\mathrm{SAD}$ shows lower levels of non-reacting compared to the healthy group. Current findings suggest that non-reacting cultivated through mindfulness is important for treating general anxiety symptoms and in clinical practice for SAD there should be enough emphasis on cultivation of non-reacting facet in this group, perhaps through targeting common symptoms such as cognitive and/or emotional reactivity to intrusive, repetitive thoughts (Desrosiers et al., 2013).

Finally, the study indicated that there was no significant difference between MDD and healthy groups in the non-reacting facet. This finding was inconsistent with our hypothesis and with previous findings in the literature (Baer et al., 2006; Desrosiers et al., 2013). Only in the longitudinal study of Raphiphatthana et al., (2016), a positive relationship between non-reacting and anhedonia in depression has been reported. The cultural differences could explain this contrast. Hence this observation suggests that in clinical practice with MDD at first, the effective cultural factors should be considered and before focusing on the process of permitting feelings and thoughts to pass through awareness without reacting to or controlling them, there may be a need to first work on increasing motivation for engaging in psychotherapy. A few limitations of this study need to be mentioned. Primarily, the cross-sectional nature of the data impedes any causal inferences. Another limitation of our study is that most of the data collection relied on self-report; therefore, scores may reflect certain self-presentation biases. In addition, further research is necessary to replicate the findings of this study, particularly for the non-reacting facet.

The results of this study suggest that non-judging, acting with awareness, and describing are the mindfulness facets that have a common role in SAD and MDD. However, it seems that observing and non-reacting facets have distinct roles in the SAD and MDD. As a result, the group with SAD had significantly higher scores in the observing facet than the MDD group, and the group with MDD had significantly higher scores in the non-reacting facet than the SAD group. Therefore, given the novelty of findings that were not significantly different between MDD and healthy groups, further research is necessary to elucidate in detail the nature of this intriguing relationships.

\section{Acknowledgments}

This research did not receive any specific grant from funding agencies in the public, commercial, or not-forprofit sectors. 


\section{Conflict of Interest}

All authors certify that this manuscript has neither been published in whole nor in part nor being considered for publication elsewhere. The authors have no conflicts of interest to declare.

\section{References}

American Psychiatric Association. (2013). Diagnostic and statistical manual of mental disorders. Washington, D.C.: American Psychiatric Association.

Baer, R. A., Smith, G. T., Hopkins, J., Krietemeyer, J., \& Toney, L. (2006). Using self-report assessment methods to explore facets of mindfulness. Assessment, 13(1), 27-45. doi $10.1177 / 1073191105283504$

Baer, R. A., Smith, G. T., Lykins, E., Button, D., Krietemeyer, J., Sauer, S., et al. (2008). Construct validity of the five facet mindfulness questionnaire in meditating and nonmeditating samples. Assessment, 15(3), 329-342. doi: $10.1177 / 1073191107313003$

Beck, A. T., Steer, R. A., \& Brown, G. K. (1996). Manual for the beck depression inventory-II. San Antonio: The Psychological Corporation

Bogaerde, A. V., Derom, E., \& De Raedt, R. (2011). Increased interoceptive awareness in fear of flying: Sensitivity to suffocation signals. Behaviour Research and Therapy, 49(6), 427-432. doi: 10.1016/j.brat.2011.03.011

Bohlmeijer, E., Prenger, R., Taal, E., \& Cuijpers, P. (2010). The effects of mindfulness-based stress reduction therapy on mental health of adults with a chronic medical disease: A metaanalysis. Journal of Psychosomatic Research, 68(6), 539-544. doi: 10.1016/j.jpsychores.2009.10.005

Brown, K. W., \& Ryan, R. M. (2004). Perils and promise in defining and measuring mindfulness: Observations from experience. Clinical Psychology: Science and Practice, 11(3), 242-248. doi: 10.1093/clipsy/bph078

Connor, K. M., Davidson, J. R., Churchill, L. E., Sherwood, A., Weisler, R. H., \& FOA, E. (2000). Psychometric properties of the social phobia inventory (SPIN): New self-rating scale. The British Journal of Psychiatry, 176(4), 379-386. doi: 10.1192/ bjp.176.4.379

Cox, B. J., Fleet, C., \& Stein, M. B. (2004). Self-criticism and social phobia in the US national comorbidity survey. Journal of Affective Disorders, 82(2), 227-234. doi: 10.1016/j.jad.2003.12.012

Dalbudak, E., Evren, C., Aldemir, S., Coskun, K. S., Yıldırım, F. G., \& Ugurlu, H. (2013). Alexithymia and personality in relation to social anxiety among university students. Psychiatry research, 209(2), 167-172. doi: 10.1016/j.psychres.2012.11.027

De Bruin, E. I., Topper, M., Muskens, J. G., Bögels, S. M., \& Kamphuis, J. H. (2012). Psychometric properties of the Five Facets Mindfulness Questionnaire (FFMQ) in a meditating and a non-meditating sample. Assessment, 19(2), 187-197. doi: $10.1177 / 1073191112446654$
Deiters, D. D., Stevens, S., Hermann, C., \& Gerlach, A. L. (2013) Internal and external attention in speech anxiety. Journal of Behavior Therapy and Experimental Psychiatry, 44(2), 143-149. doi: 10.1016/j.jbtep.2012.09.001

Desrosiers, A., Klemanski, D. H., \& Nolen-Hoeksema, S. (2013). Mapping mindfulness facets onto dimensions of anxiety and depression. Behavior Therapy, 44(3), 373-384. doi: 10.1016/j. beth.2013.02.001

Dogaheh, E. R. (2013). Psychometric Properties of Farsi Version of the Social Phobia Inventory (SPIN). Procedia-Social and Behavioral Sciences, 84, 763-768. doi: 10.1016/j.sbspro.2013.06.642

Garland, E. L., Farb, N. A., R. Goldin, P., \& Fredrickson, B. L. (2015). Mindfulness broadens awareness and builds eudaimonic meaning: A process model of mindful positive emotion regulation. Psychological Inquiry, 26(4), 293-314. doi: 10.1080/1047840x.2015.1064294

Grant, B. F., Hasin, D. S., Blanco, C., Stinson, F. S., Chou, S. P., Goldstein, R. B., et al. (2005). The Epidemiology of social anxiety disorder in the United States: Results from the national epidemiologic survey on alcohol and related conditions. Journal of Clinical Psychiatry, 66(11), 1351-61. doi: 10.4088/jcp. v66n1102

Grant, D. M., Judah, M. R., Mills, A. C., Lechner, W. V., Davidson, C. L., \& Wingate, L. R. (2014). Rumination and excessive reassurance seeking: mediators of the relationship between social anxiety and depression? Journal of Psychopathology and Behavioral Assessment, 36(3), 465-474. doi: 10.1007/s10862-0139399-5

Gross, J. J. (2014). Handbook of emotion regulation. New York: Guilford Press.

Helbig-Lang, S., Poels, V., \& Lincoln, T. M. (2016). Performance perceptions and self-focused attention predict post-event processing after a real-life social performance situation. Anxiety, Stress, \& Coping, 29(6), 708-715. doi: 10.1080/10615806.2016.1157168

Hölzel, B. K., Carmody, J., Vangel, M., Congleton, C., Yerramsetti, S. M., Gard, T., et al. (2011). Mindfulness practice leads to increases in regional brain gray matter density. Psychiatry Research: Neuroimaging, 191(1), 36-43. doi: 10.1016/j.pscychresns.2010.08.006

Hsu, K. J., Beard, C., Rifkin, L., Dillon, D. G., Pizzagalli, D. A., \& Björgvinsson, T. (2015). Transdiagnostic mechanisms in depression and anxiety: The role of rumination and attentional control. Affective Disorders, 188, 22-27. doi: 10.1016/j. jad.2015.08.008

Kocovski, N. L., Fleming, J. E., Hawley, L. L., Ho, M. H. R., \& Antony, M. M. (2015). Mindfulness and acceptance-based group therapy and traditional cognitive behavioral group therapy for social anxiety disorder: Mechanisms of change. Behaviou Research and Therapy, 70, 11-22. doi: 10.1016/j.brat.2015.04.005

Koyuncu, A., Ertekin, E., Ertekin, B. A., Binbay, Z., Yüksel, Ç., Deveci, E., et al. (2015). Relationship between atypical depression and social anxiety disorder. Psychiatry Research, 225(1-2), 79-84. doi: 10.1016/j.psychres.2014.10.014

Mor, N., \& Winquist, J. (2002). Self-focused attention and negative affect: A meta-analysis. Psychological Bulletin, 128(4), 63862. doi: $10.1037 / / 0033-2909.128 .4 .638$ 
Mor, N., Doane, L. D., Adam, E. K., Mineka, S., Zinbarg, R. E., Griffith, J. W., et al. (2010). Within-person variations in selffocused attention and negative affect in depression and anxiety: A diary study. Cognition and Emotion, 24(1), 48-62. doi: $10.1080 / 02699930802499715$

Nowakowski, M. E., McFarlane, T., \& Cassin, S. (2013). Alexithymia and eating disorders: A critical review of the literature. Journal of Eating Disorders, 1(1), 21. doi: 10.1186/2050-2974-121

Ohayon, M. M., \& Schatzberg, A. F. (2010). Social phobia and depression: Prevalence and comorbidity. Journal of Psychosomatic Research, 68(3), 235-243. doi: 10.1016/j.jpsychores.2009.07.018

Ranta, K., La Greca, A. M., Kaltiala-Heino, R., \& Marttunen, M. (2016). Social Phobia and Educational and Interpersonal Impairments in Adolescence: A Prospective Study. Child Psychiatry \& Human Development, 47(4), 665-677. doi: 10.1007/ s10578-015-0600-9

Raphiphatthana, B., Jose, P. E., \& Kielpikowski, M. (2016). How do the facets of mindfulness predict the constructs of depression and anxiety as seen through the lens of the tripartite theory? Personality and Individual Differences, 93, 104-111. doi: 10.1016/j.paid.2015.08.005

Rubio, J. M., Markowitz, J. C., Alegría, A., Pérez-Fuentes, G, Liu, S. M., Lin, K. H., et al. (2011). Epidemiology of chronic and nonchronic major depressive disorder: results from the national epidemiologic survey on alcohol and related conditions. Depression and Anxiety, 28(8), 622-631. doi: 10.1002/ da.20864

Schuver, K. J., \& Lewis, B. A. (2016). Mindfulness-based yoga intervention for women with depression. Complementary Therapies in Medicine, 26, 85-91. doi: 10.1016/j.ctim.2016.03.003

Taylor, S., Abramowitz, J. S., \& McKay, D. (2012). Non-adherence and non-response in the treatment of anxiety disorders. Journal of Anxiety Disorders, 26(5), 583-589. doi: 10.1016/j. janxdis.2012.02.010

Washburn, D., Wilson, G., Roes, M., Rnic, K., \& Harkness, K. L. (2016). Theory of mind in social anxiety disorder, depression, and comorbid conditions. Journal of Anxiety Disorders, 37, 7177. doi: 10.1016/j.janxdis.2015.11.004 